\title{
ES Research Square \\ Medication Overuse Withdrawal in Children and Adolescents does not Always Improve Headache: A Cross-Sectional Study
}

Romina Moavero ( $\square$ rominamoavero@hotmail.com )

Universita degli Studi di Roma Tor Vergata Dipartimento di Medicina dei Sistemi https://orcid.org/0000-0002-6200-060X

Maddalena Stornelli

Universita degli Studi di Roma Tor Vergata Dipartimento di Medicina dei Sistemi

Laura Papetti

Ospedale Pediatrico Bambino Gesu

Fabiana Ursitti

Ospedale Pediatrico Bambino Gesu

Michela Ada Noris Ferilli

Ospedale Pediatrico Bambino Gesu

Martina Balestri

Ospedale Pediatrico Bambino Gesu

Giorgia Sforza

Universita degli Studi di Roma Tor Vergata Dipartimento di Medicina dei Sistemi

Samuela Tarantino

Ospedale Pediatrico Bambino Gesu

Federico Vigevano

Ospedale Pediatrico Bambino Gesu

Massimiliano Valeriani

Ospedale Pediatrico Bambino Gesu

\section{Research article}

Keywords: . chronic migraine, medication overuse headache, children, ICHD-3 criteria, secondary headache, treatment

Posted Date: May 28th, 2020

DOI: https://doi.org/10.21203/rs.3.rs-31087/v1 
License: (c) (i) This work is licensed under a Creative Commons Attribution 4.0 International License. Read Full License 


\section{Abstract}

Background. $\mathrm{MOH}$ can be diagnosed in subjects with headache occurring 15 days/month in association with a regular medication overuse, but its existence is not universally accepted. ICHD-3 redefined criteria for $\mathrm{MOH}$, removing the criterion associating drug suspension with headache course. Aim of our study was to compare the rate of patients diagnosed with medication overuse headache $(\mathrm{MOH})$ according to ICHD-2 and ICHD-3 criteria, to verify the degree of concordance. Secondary aim was to verify if drug withdrawal was really associated with pain relief.

Methods. In this cross-sectional study, we retrospectively analyzed a sample of 400 patients followed for primary chronic headache at the Headache Center of Bambino Gesù Children's Hospital. We then selected those presenting with a history of medication overuse, and we applied both ICHD-2 and ICHD-3 criteria to verify in which patients the criteria would identify a clinical diagnosis of $\mathrm{MOH}$.

Results. We identified 42 subjects (10.5\%) with $\mathrm{MOH} ; 23$ of them (55\%) presented a relief of headache withdrawing drug overuse. Regarding the applicability of the ICHD-2 criteria, $43 \%$ of patients $(18 / 42)$ fulfilled all criteria, while all ICHD-3 diagnostic criteria were satisfied in $76 \%$ of patients (32/42). Eighteen patients (43\%) satisfied both ICHD-2 and ICHD-3 criteria, while 10 patients (24\%) did not satisfy either diagnostic criteria.

Conclusions. Our study suggests that in children and adolescents withdrawing medication overuse is not always associated with a clinical benefit. Therefore, though allowing a $\mathrm{MOH}$ diagnosis in a higher rate of patients as compared to ICHD-2, the application of ICHD-3 criteria does not guarantee a true a causal relationship between medication overuse and headache worsening.

\section{Introduction}

Medication overuse headache $(\mathrm{MOH})$ is a disabling disorder occurring when a subject with a primary headache disorder begins to suffer from a chronic headache, presumably due to a too frequent use of drugs usually assumed to extinguish pain. The term "medication-overuse headache" was first used in the second edition of the ICHD ${ }^{[1]}$ although a "drug-induced headache" was previously described in its first edition. In the last edition of International Classification of Headache Disorder, ${ }^{[2]} \mathrm{MOH}$ is listed as a secondary headache, in the section focused on "Headache attributed to a substance or its withdrawal".

Although pathophysiologic mechanisms of $\mathrm{MOH}$ are still largely unclear, a genetic predisposition likely plays an important role. ${ }^{[3,4]}$ Another potentially significant pathogenetic factor taken into consideration is the interaction between drugs used and neurotransmitters ${ }^{[5]}$ and/or hormonal systems. ${ }^{[6]}$ Other factors investigated over time include the presence of abnormal neuronal excitability ${ }^{[7]}$ and changes in grey matter volumes ${ }^{[8]}$ and cerebral metabolism. ${ }^{[9-11]}$

The overall prevalence of $\mathrm{MOH}$ in the general population is $0.5-2.6 \%$, although it varies between different studies, probably as a consequence of different diagnostic criteria published over time and different 
methods used to collect epidemiological data. ${ }^{[11,12]}$ Very few epidemiological studies are available in pediatric population. Data from Norway and Taiwan report prevalence rate of $0.2 \%$ and $0.3 \%$ respectively. $[13,14]$ Data from pediatric populations with chronic primary headache disorders report a medication overuse in $10-60 \%$ of cases. ${ }^{[15]}$ Both in adults and children, $\mathrm{MOH}$ appears to be more common among females than males. ${ }^{[16,17]}$

Clinical features are usually the same of preexisting primary headache disorder. ${ }^{[11]}$ In pediatric patients, it is more commonly associated with chronic migraine (CM). ${ }^{[18]}$ Non-steroid anti-inflammatory drugs (NSAIDs) are the class of drugs more often overused, followed by paracetamol and triptans. ${ }^{[16]}$ Historically, the treatment of $\mathrm{MOH}$ includes two main strategies: a detoxification program with discontinuation of drugs overused, and initiation of pharmacological and nonpharmacological preventive therapy. ${ }^{[11]}$

In the last two decades, diagnostic criteria for $\mathrm{MOH}$ were gradually changed. Initially, $\mathrm{MOH}$ could be diagnosed only if the headache resolved or reverted to the previous pattern within 2 months after withdrawal of the overused medication. ${ }^{[19]}$ In the revision of diagnostic criteria published in $2006,{ }^{[20]}$ the Headache Classification Committee proposed to remove the criterion concerning the effect of drug suspension on headache course, and this modification was kept in the last published version of ICHD-3 (Table 1) ${ }^{[2]}$

Table 1

Diagnostic criteria for MOH by ICHD-2 (2004) ${ }^{[1]}$ and by ICHD-3 (2018) ${ }^{[2]}$.

\section{ICHD-2 \\ ICHD-3}

A.Headache present on $\geq 15$ days/month fulfilling criteria $C$ and $D$

B.Regular overuse2 for $\geq 3$ months of one or more drugs that can be taken for acute and/or symptomatic treatment of headache

C.Headache has developed or markedly worsened during medication overuse

D.Headache resolves or reverts to its previous pattern within 2 months after discontinuation of overused medication
A. Headache occurring on $\geq 15$ days/month in a patient with a pre-existing headache disorder

B. Regular overuse for $>3$ months of one or more drugs that can be taken for acute and/or symptomatic treatment of headache

C. Not better accounted for by another ICHD-3 diagnosis.

Therefore, $\mathrm{MOH}$ can be presently diagnosed in a subject with a history of a preexisting primary headache, presenting with headache occurring 15 days per month in association with a regular medication use exceeding specific thresholds.

A direct consequence of new criteria could be an increase of definite diagnosis, since $\mathrm{MOH}$ can now be diagnosed even in the absence of improvement after drugs withdrawal. However, diagnostic criteria and even the existence of this specific nosographic entity are not universally accepted. For instance, some 
authors wondered whether medication overuse is the real cause of headache in all subjects fulfilling diagnostic criteria for $\mathrm{MOH} .{ }^{[15,19,21]}$ Indeed, in some individuals medication overuse can increase headache frequency, and discontinuing the medications can have a benefit, but this is not the case in all individuals overusing medications. In some case, increasing headache frequency represents a worsening of the primary headache disorder, and increased use of acute medications is its consequence. ${ }^{[15]}$

Aim of our study was to compare the rate of patients diagnosed with $\mathrm{MOH}$ according to the old ICHD-2 and new ICHD-3 criteria, in order to verify the degree of concordance and understand if the new classification really led to different diagnostic rates. Secondary aim was to verify if drug withdrawal is really associated with pain relief, and therefore to investigate in a large sample of pediatric patients whether $\mathrm{MOH}$ is a true entity.

\section{Materials And Methods}

In this cross-sectional study, we retrospectively analyzed a sample of patients followed at the Headache Center of the Neuroscience Department of Bambino Gesù Pediatric Hospital in Rome. We included all patients with chronic headache, diagnosed according to the ICHD-3 criteria ${ }^{[2]}$ and followed-up at our Headache Center in the period 2010-2018, whose parents gave their informed consent to be contacted for retrospective studies. The sample was partially published in Papetti et al, 2019. ${ }^{[18]}$ In particular, 210 out of 377 patients included in the Papetti et al.'s sample (collected between 2010 and 2016) were considered for the present study while the remaining 190 patients were totally original. Moreover, only 20 out 42 of the $\mathrm{MOH}$ patients were issued from the Papetti et al. population, while the remaining 22 patients are totally original. As compared to Papetti et al., the present study investigated different points: 1) the comparison of the applicability of the ICHD-2 and ICHD-3 criteria of pediatric $\mathrm{MOH}$ patients, and 2) the clinical outcome after medication withdrawal in $\mathrm{MOH}$ children and adolescents.

Among these patients, we selected those presenting with a personal history of medication overuse, defined as regular use of abortive therapy: at least 10 days per month for ergotamine, triptans, opioids or combination-analgesic medication and 15 or more days per month for non-opioid analgesics (paracetamol, non-steroidal anti-inflammatory drug or acetylsalicylic acid). Overuse should have been carried on for at least 3 months. In all patients, the clinical diagnosis of $\mathrm{MOH}$ was tested according to either ICHD-2 or ICHD-3 version criteria, in order to verify the degree of concordance. The diagnoses were made independently by two experienced neurologists, blinded to each other's rating (MV, LP). All these data were initially extrapolated by clinical charts, and then confirmed and deeply investigated during follow-up visits and/or telephonic interviews.

Clinical data collected for each patient were: the pre-existing primary headache type, the clinical characteristics of headache and other symptoms associated, and the treatment used, both symptomatic and prophylactic. 
The usual therapeutic strategy was an intensive verbal advice to discontinue the medication overuse, with the suggestion of a different symptomatic treatment than the overused one. In almost all cases, a preventive medication was also proposed, at this same time. Medication withdrawal was considered successful if criteria for overuse were no more satisfied, and it was conducted over a 2 months period. The outcome of medication withdrawal was assessed after two additional months of follow-up, and it was considered effective if chronic headache reverted to episodic.

Ethical Board approval for retrospective study was obtained.

\section{Statistical analysis}

Statistical analysis was conducted by SPSS version 22.0. To test the hypothesis of a possible association between response to medication withdrawal and sex, type of overused medication and preventive treatment we used the $\chi 2$ test. A $p$-value of $\leq 0.05$ was considered significant.

Furthermore, a multiple regression logistic analysis has been used to evaluate whether age, age at first attack (0-6 years, 7-10 years, 11-14 years, 15-18 years), or type of preventive treatment (topiramate, 5hydroxytriptophane, flunarizine, amitryptiline) influenced response to withdrawing overused medication. Response to medication withdrawal was selected as a dependent variable, and then all the other variables have been tested as independent variables in a block entry to evaluate the $t$ value, the significance, the standard error and the upper and lower limit in a confidence interval of $95 \%$.

\section{Results}

We collected and analyzed clinical data from a sample of 400 patients ( $134 \mathrm{M}, 266 \mathrm{~F}$ ) with primary chronic headache. There were no missing data in our sample. Seventy-five percent of patients presented with $\mathrm{CM}, 13 \%$ with chronic tension-type headache, and $12 \%$ with New Daily-Persistent Headache (NDPH). In $11 \%$ of patients (10 patients with NDPH and 37 with $\mathrm{CM}$ ) migraine with aura (Mwa) was diagnosed.

In this sample we identified 42 subjects $(10.5 \%$, Table 2$)$ with symptomatic medication overuse defined as above (at least 10 days per month for ergotamine, triptans, opioids and 15 or more days per month for non-opioid analgesics). The sample was mainly composed by females $(11 \mathrm{M}, 26 \%-31 \mathrm{~F}, 74 \%)$, with a mean age of 13 years at their first medical examination (range: 8-17 years). All patients (100\%) presented CM, and 9\% (4/42) presented also Mwa. The age at onset of headache was $\leq 6$ years in $9 \%$ of patients (4/42), 7-10 years in 29\% (12/42), 11-14 years in 48\% (20/42), $15-18$ in $14 \%(6 / 42)$. Mean duration of mediation overuse was 4.1 months (range $3-6$ months) 
Table 2

Demographic features of patients with Chronic Migraine and medication overuse.

\begin{tabular}{|c|c|c|}
\hline Patients. & $\begin{array}{l}N \\
42\end{array}$ & $\begin{array}{l}\% \\
100\end{array}$ \\
\hline Mean age: 13 years (range 8-17 years) & - & - \\
\hline Sex. & 11 & 26 \\
\hline $\begin{array}{l}\cdot \text { Males } \\
\cdot \text { Fermales }\end{array}$ & 31 & 74 \\
\hline $\begin{array}{l}\text { Diagnosis: } \\
\cdot \text { Chronic Migraine } \\
\cdot \text { Migraine with aura }\end{array}$ & $\begin{array}{l}42 \\
4\end{array}$ & $\begin{array}{l}100 \\
9\end{array}$ \\
\hline Age at onset. & 4 & 9 \\
\hline$\cdot<6$ years & 12 & 29 \\
\hline$\cdot 7-10$ years & 20 & 48 \\
\hline $\begin{array}{l}\cdot 11-14 \text { years } \\
\cdot 15-18 \text { years }\end{array}$ & 6 & 14 \\
\hline Symptoms associated: & 34 & 81 \\
\hline - Photophobia & 34 & 81 \\
\hline · Phonophobia & 30 & 71 \\
\hline $\begin{array}{l}\cdot \text { Nausea and vomit } \\
\cdot \text { Dizzines }\end{array}$ & 18 & 42 \\
\hline Symptomatic Treatment. & 42 & 100 \\
\hline$\cdot N S A I D s$ & 42 & 100 \\
\hline - Triptans & 9 & 21 \\
\hline Prophylactic Treatment. & 39 & 93 \\
\hline - Amitriptyline & 31 & 79 \\
\hline - Topiramate & 15 & 38 \\
\hline - Flunarizine & 11 & 28 \\
\hline - Tryptophan & 6 & 15 \\
\hline
\end{tabular}


Photophobia and phonophobia were both present in $81 \%$ of patients (34/42), nausea and vomiting in $71 \%$ (30/42) and dizziness in $42 \%$ (18/42). All patients used NSAIDs as symptomatic treatment; $21 \%$ of the sample (9/42) used triptans as further option after a poor response to NSAIDs. Moreover, prophylactic treatment was prescribed in 93\% (39/42) of patients, including drug-naïve patients and those who were assuming an ineffective prophylactic therapy. Amitriptyline was the most used drug (79\%, 33/42); topiramate was used in $38 \%(16 / 42)$, flunarizine in $28 \%(12 / 42)$ and tryptophan in $15 \%(6 / 42)$. More than one type of prophylactic drug was used in $28 \%$ of the sample $(12 / 42$; these patients were already assuming one prophylactic drug at the time of our first visit). After withdrawing symptomatic drugs overuse, a clear benefit was evident only in $23 / 42$ subjects ( $55 \%$ ).

Regarding the applicability of the ICHD-2 criteria, $43 \%$ of patients (18/42) fulfilled diagnosis of $\mathrm{MOH}$ while $57 \%$ (24/42) did not fulfill all the diagnostic criteria (Fig. 1). In detail, 21/42 patients (50\%) fulfilled criterion A; 35/42 (83\%) criterion B, 37/42 (88\%) criterion C, and 23/42 (55\%) criterion D (Fig. 2A).

On the other hand, all ICHD-3 diagnostic criteria were fulfilled in $76 \%$ of patients (32/42, Fig. 1). Specifically, ICHD-3 criterion A was fulfilled by $40 / 42$ patients (95\%), criterion B by $35 / 42$ (83\%), and criterion C by $40 / 42$ (95\%) (Fig. 2B).

Eighteen patients (43\%) satisfied both ICHD-2 and ICHD-3 criteria, while 10 patients (24\%) did not satisfy either diagnostic criteria.

None of the analyzed variables (age at evaluation, age at first attack, or type of preventive treatment) showed a statistical significance at the multivariate analysis (table 3). The improvement after drug overuse withdrawal was observed in $20 / 31(65 \%)$ of the females of our sample, compared with $3 / 8(38 \%)$ of males $(p=0.03)$. However, we have to underline that our sample was mainly composed by females. The type of overused drug was not associated to response to withdrawal $(p=0.93)$. 
Table 3

Results of multiple regression logistic analysis: beta coefficients and significance, with lower and upper limits at 95\% confidence interval

\begin{tabular}{|llll|}
\hline & $\begin{array}{l}\text { b coefficient } \pm \text { standard } \\
\text { error }\end{array}$ & Significance & $\begin{array}{l}\text { Confidence interval } \\
\text { (95\%) }\end{array}$ \\
\hline Age & $0.001 \pm 0.004$ & 0.882 & $-0.007 ; 0.009$ \\
\hline $\begin{array}{l}\text { Age at migraine onset } 0- \\
6 \text { years }\end{array}$ & $-0.483 \pm 0.375$ & 0.207 & $-1.246 ; 0.280$ \\
\hline $\begin{array}{l}\text { Age at migraine onset 7- } \\
\text { 10 years }\end{array}$ & $-0.246 \pm 0.232$ & 0.295 & $-0.718 ; 0.225$ \\
\hline $\begin{array}{l}\text { Age at migraine onset 11- } \\
\text { 14 years }\end{array}$ & $-0.022 \pm 0.155$ & 0.213 & $-0.117 ; 0.508$ \\
\hline $\begin{array}{l}\text { Age at migraine onset 15- } \\
\text { 18 years }\end{array}$ & $-0.005 \pm 0.262$ & 0.934 & $-0.554 ; 0.511$ \\
\hline $\begin{array}{l}\text { Topiramate } \\
\text { Flunarizine }\end{array}$ & $-0.005 \pm 0.207$ & 0.981 & $-0.427 ; 0.416$ \\
\hline TriptOH & $0.065 \pm 0.236$ & 0.785 & $-0.415 ; 0.545$ \\
\hline Amitriptiline & $-0.339 \pm 0.273$ & 0.223 & $-0.895 ; 0.217$ \\
\hline
\end{tabular}

\section{Discussion}

Our retrospective study on a large sample of pediatric patients revealed that the application of ICHD-3 criteria allows a $\mathrm{MOH}$ diagnosis in a higher rate of patients (76\% versus $43 \%$ ), thus proving more sensitive than ICHD-2 criteria. The main difference between two versions is that ICHD-3 criteria do not require remission or improvement of headache after the regular drugs overuse is stopped. However, ICHD3 version, removing the relationship between pain and drug overuse, seems to consider the $\mathrm{MOH}$ as a fully established diagnosis, while it is still matter of debate.

A second crucial finding of our study is that in our sample symptomatic drug withdrawal was not always sufficient to revert chronic to episodic migraine, thus strengthening the concept that, in turn, medication overuse was probably not sufficient to make our patients' migraine become chronic. Specifically, in our sample, medication withdrawal did not cause any reduction in headache frequency in almost half of patients (45\%). Furthermore, 22/23 patients (95\%) showing an improvement of symptoms after drugs withdrawal (meaning a return to episodic headache) were assuming a preventive therapy at the same time. Therefore, it is very difficult to judge if the positive effect on headache frequency was caused by one or the other therapeutic approach used.

The few studies published on $\mathrm{MOH}$ in pediatric age show a response rate to drug withdrawal (defined as a reduction more than $50 \%$ of headache frequency) between $40-77 \%{ }^{[3,22-24]}$ On the other hand, a lack of 
improvement after drug withdrawal is reported in $4-41 \%$ of patients (Table 3 ). A genetic study on a pediatric population with $\mathrm{CM}$ and medication overuse, identified statistically significant gene expression differences between responders and non-responders to withdrawal, thus suggesting a possible biomarker to distinguish true $\mathrm{MOH}$ patients from chronic migraineurs in whom overused medication does not have a pathophysiological role. ${ }^{[3]}$

Considering also $\mathrm{MOH}$ studies in adults, we found limited evidence supporting a clear benefit of discontinuation of symptomatic medications without concomitant introduction of a preventive therapy. [25] In particular, clear clinical benefits after only withdrawing overused medication have been described in less than one third of reported patients. ${ }^{[26-28]}$ Another important bias of the available studies is represented by patients who pretend to have withdrawn symptomatic treatment, while keeping overusing drugs. Furthermore, randomized controlled clinical trial investigating topiramate ${ }^{[29,30]}$ and onabotulinumtoxin ${ }^{[31,32]}$ for treatment of $\mathrm{CM}$ showed that immediate initiation of preventive treatment without early suspension of the overused medication is effective in patients with $\mathrm{CM}$ and medication overuse. ${ }^{[33]}$ Most of these studies also lacked an adequate control group, thus making it impossible to differentiate patients presenting a benefit due to the typical cyclic pattern of headache, and those really responding to overuse cessation. Some authors hypothesized that medication overuse can be seen as an epiphenomenon of a chronic headache presenting with periods of higher frequency and severity, ${ }_{1}^{[19]}$ thus suggesting that a combined strategy of preventive therapy and overuse cessation could be more appropriate. Indeed, a recent review of the available literature data concluded that the combined approach of discontinuation of overused acute medications and a concurrent preventive intervention should be the standard of care, ${ }^{[25]}$ as already recommended by EFNS (European Federation of Neurological Societies) guidelines for $\mathrm{MOH}^{[34]}$

\section{Limitations of the study}

Our study certainly presents some limitations. First of all, the retrospective nature of the study is a limitation in itself. Furthermore, our population might not be representative of the general population, as patients have been recruited in a tertiary headache center. However, it is also important to underline that patients sufferings from chronic headache and medication overuse usually refer to tertiary centers, and therefore our sample might be overlapping to general pediatric $\mathrm{MOH}$ samples. Lastly, the information of drug use is based on patients' diary, and, especially in case of adolescents, these data might not always be completely reliable.

\section{Conclusions}

In conclusion, our data on a large pediatric population of subjects with chronic headache and medication overuse, show that withdrawing medication overuse is not always associated with a clinical benefit. This means that a causal relationship between medication overuse and headache worsening is not always demonstrable, thus suggesting that the concept of $\mathrm{MOH}$ might be not universally applicable. Although 
ICHD-3 criteria for $\mathrm{MOH}$ appear to be more sensitive than ICHD-2, allowing a definite diagnosis in a higher number of patients, they do not contribute to make this issue less puzzling, since the new ICHD version considers $\mathrm{MOH}$ as a definite nosographic entity, which is not supported by the present literature. In other words, if the effect of drug suspension on headache course is not verified, a sure relationship between medication overuse and headache chronification cannot be demonstrated in all patients. A proposal for a new systematic review on pediatric $\mathrm{MOH}$ has been recently published ${ }^{[35]}$ and will hopefully contribute to clarify this issue.

\section{Abbreviations}

$\mathrm{CM}$

Chronic Migraine

EFNS

European Federation of Neurological Societies

ICHD

International Classification of Headache Disorders

$\mathrm{MOH}$

Medication Overuse Headache

Mwa

migraine with aura

$\mathrm{NDPH}$

New Daily-Persistent Headache

NSAIDs

Non-steroid anti-inflammatory drugs

\section{Declarations}

\section{Ethics approval and consent to participate}

We obtained approval from the Ethical Board of our Hospital, and patients and/or caregivers gave their consent to participate to this retrospective study.

\section{Consent for publication}

Not applicable

\section{Availability of data and material}

The datasets generated and/or analysed during the current study are not publicly available due to privacy reasons

Funding 
This study has no external funding source

\section{Conflict of interest}

The Authors declare that they have no conflict of interest

\section{Authors' Contribution}

RM, LP and MV conceptualized the study. RM, LP, MS inserted data in the database and analyzed data. LP, MS, FU, MANF, MB, GS, MV followed up all patients included in the study. RM drafted the manuscript. FV and MV revised the different versions of the manuscript. All the Authors revised and approved the final version of the manuscript.

\section{Acknowledgements}

We would like to thank Pierfrancesco Alaimo di Loro for his help in performing statistical analysis.

\section{References}

1. Headache Classification Subcommittee of the International Headache S. The International Classification of Headache Disorders: 2nd edition. Cephalalgia. 2004;24 Suppl 1:9-160

2. Headache Classification Committee of the International Headache Society (IHS) The International Classification of Headache Disorders, 3rd edition. Cephalalgia. 2018 Jan;38(1):1-211

3. Hershey AD, Burdine D, Kabbouche MA et al (2011 Jan) Genomic expression patterns in medication overuse headaches. Cephalalgia 31(2):161-171

4. Cargnin S, Viana M, Ghiotto $\mathrm{N}$ et al (2014 Jul) Functional polymorphisms in COMT and SLC6A4 genes influence the prognosis of patients with medication overuse headache after withdrawal therapy. Eur J Neurol 21(7):989-995

5. Rossi C, Pini LA, Cupini ML et al. Endocannabinoids in platelets of chronic migraine patients and medication-overuse headache patients: relation with serotonin levels. Eur J Clin Pharmacol. 2008 Jan;64(1):1-8

6. Rainero I, Ferrero M, Rubino E et al. Endocrine function is altered in chronic migraine patients with medication-overuse. Headache. 2006 Apr;46(4):597-603

7. Ferraro D, Vollono C, Miliucci R et al (2012 May) Habituation to pain in "medication overuse headache": a CO2 laser-evoked potential study. Headache 52(5):792-807

8. Riederer F, Gantenbein AR, Marti M et al (2013 Sep) Decrease of gray matter volume in the midbrain is associated with treatment response in medication-overuse headache: possible influence of orbitofrontal cortex. J Neurosci 25(39):15343-15349 33(

9. Fumal A, Laureys S, Di Clemente L et al (2006 Feb) Orbitofrontal cortex involvement in chronic analgesic-overuse headache evolving from episodic migraine. Brain 129(Pt 2):543-550 
10. Ferraro S, Grazzi L, Muffatti R et al (2012 Nov-Dec) In medication-overuse headache, fMRI shows long-lasting dysfunction in midbrain areas. Headache 52(10):1520-1534

11. Diener HC, Holle D, Solbach K et al (2016 Oct) Medication-overuse headache: risk factors, pathophysiology and management. Nat Rev Neurol 12(10):575-583

12. Westergaard ML, Hansen EH, Glumer C et al (2014 May) Definitions of medication-overuse headache in population-based studies and their implications on prevalence estimates: a systematic review. Cephalalgia 34(6):409-425

13. Dyb G, Holmen TL, Zwart JA. Analgesic overuse among adolescents with headache: the Head-HUNTYouth Study. Neurology. 2006 Jan 24;66(2):198-201

14. Wang SJ, Fuh JL, Lu SR et al. Chronic daily headache in adolescents: prevalence, impact, and medication overuse. Neurology. 2006 Jan 24;66(2):193-7

15. Gelfand AA, Goadsby PJ (2014 Jul) Medication overuse in children and adolescents. Curr Pain Headache Rep 18(7):428

16. Pakalnis A, Butz C, Splaingard D et al (2007 Dec) Emotional problems and prevalence of medication overuse in pediatric chronic daily headache. J Child Neurol 22(12):1356-1359

17. Pakalnis A, Kring D (2012 May) Chronic daily headache, medication overuse, and obesity in children and adolescents. J Child Neurol 27(5):577-580

18. Papetti L, Salfa I, Battan B et al (2019) Features of Primary Chronic Headache in Children and Adolescents and Validity of Ichd 3 Criteria. Front Neurol 10:92

19. Vandenbussche $\mathrm{N}$, Laterza D, Lisicki $\mathrm{M}$ et al. Medication-overuse headache: a widely recognized entity amidst ongoing debate. J Headache Pain. 2018 Jul 13;19(1):50

20. Headache Classification C, Olesen J, Bousser MG et al (2006 Jun) New appendix criteria open for a broader concept of chronic migraine. Cephalalgia 26(6):742-746

21. Scher Al, Rizzoli PB, Loder EW. Medication overuse headache: An entrenched idea in need of scrutiny. Neurology. 2017 Sep 19;89(12):1296-1304

22. Hering-Hanit R, Gadoth N, Cohen A et al (2001 Jun) Successful withdrawal from analgesic abuse in a group of youngsters with chronic daily headache. J Child Neurol 16(6):448-449

23. Wiendels NJ, van der Geest MC, Neven AK et al (2005 Jun) Chronic daily headache in children and adolescents. Headache 45(6):678-683

24. Kossoff EH, Mankad DN (2006 Jan) Medication-overuse headache in children: is initial preventive therapy necessary? J Child Neurol 21(1):45-48

25. Chiang CC, Schwedt TJ, Wang SJ et al. Treatment of medication-overuse headache: A systematic review. Cephalalgia. 2016 Apr;36(4):371-86

26. Zeeberg P, Olesen J, Jensen R. Probable medication-overuse headache: the effect of a 2-month drugfree period. Neurology. 2006 Jun 27;66(12):1894-8

27. Hagen K, Albretsen C, Vilming ST et al (2009 Feb) Management of medication overuse headache: 1year randomized multicentre open-label trial. Cephalalgia 29(2):221-232 
28. Sarchielli P, Messina P, Cupini LM et al (2014 Aug) Sodium valproate in migraine without aura and medication overuse headache: a randomized controlled trial. Eur Neuropsychopharmacol 24(8):1289-1297

29. Diener HC, Bussone G, Van Oene JC et al (2007 Jul) Topiramate reduces headache days in chronic migraine: a randomized, double-blind, placebo-controlled study. Cephalalgia 27(7):814-823

30. Silberstein SD, Lipton RB, Dodick DW et al (2007 Feb) Efficacy and safety of topiramate for the treatment of chronic migraine: a randomized, double-blind, placebo-controlled trial. Headache 47(2):170-180

31. Aurora SK, Dodick DW, Turkel CC et al (2010 Jul) OnabotulinumtoxinA for treatment of chronic migraine: results from the double-blind, randomized, placebo-controlled phase of the PREEMPT 1 trial. Cephalalgia 30(7):793-803

32. Diener HC, Dodick DW, Aurora SK et al (2010 Jul) OnabotulinumtoxinA for treatment of chronic migraine: results from the double-blind, randomized, placebo-controlled phase of the PREEMPT 2 trial. Cephalalgia 30(7):804-814

33. Diener HC. Detoxification for medication overuse headache is not necessary. Cephalalgia. 2012 Apr;32(5):423-7

34. Evers S, Jensen R (2011 Sep) European Federation of Neurological S. Treatment of medication overuse headache-guideline of the EFNS headache panel. Eur J Neurol 18(9):1115-1121

35. VanderPluym J, Gautreaux J, Burch R et al (2020 Jan) Evidence Regarding Medication Overuse Headache in Children and Adolescents: Protocol for a Systematic Review. Headache 60(1):171-177

\section{Figures}
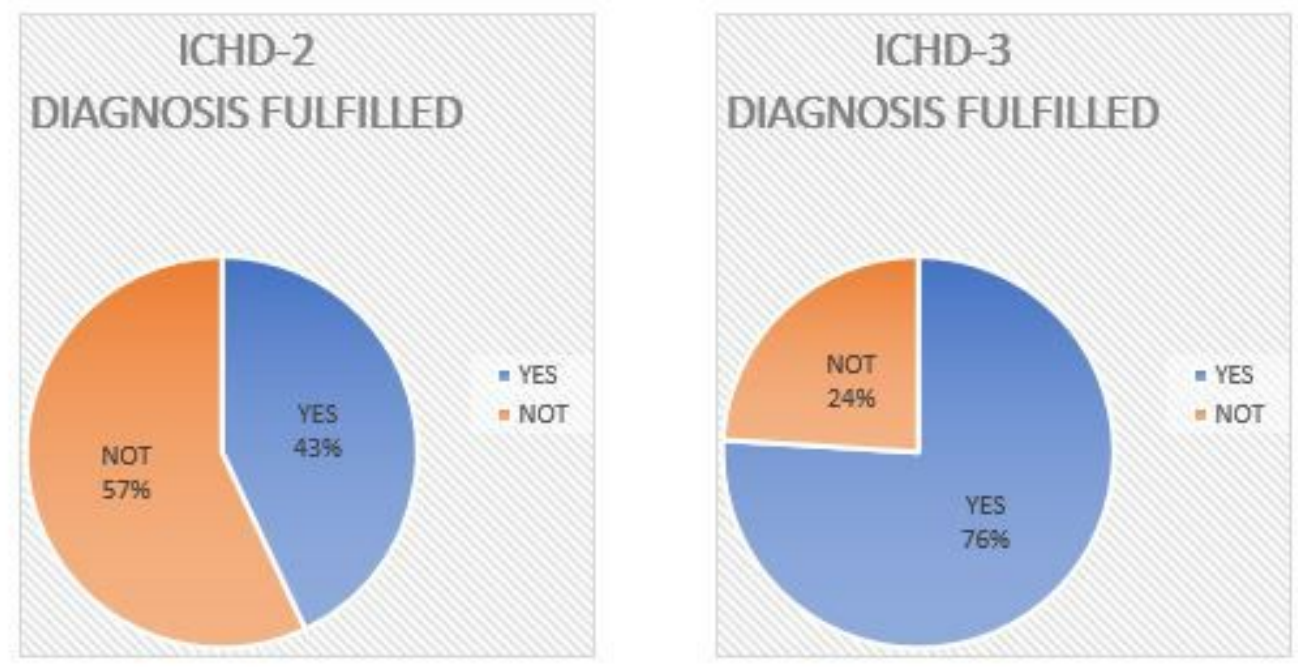

\section{Figure 1}


Different diagnostic rate of Medication Overuse Headache according to ICHD-2 criteria (figure 1A) versus ICHD-3 criteria (figure 1B).

\section{APPLICABILITY OF ICHD-2 CRITERIA}

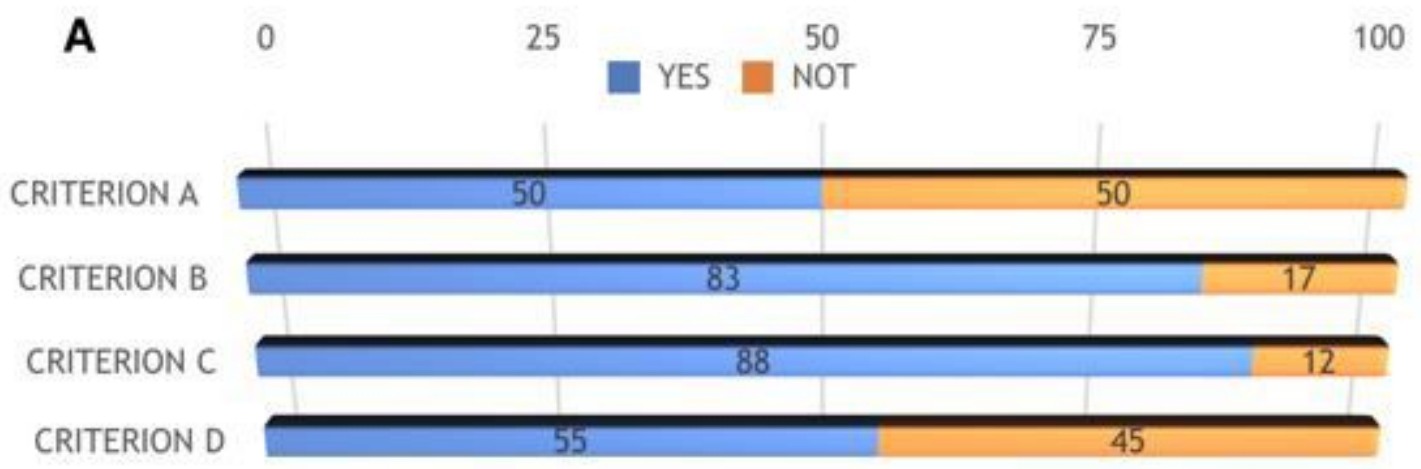

\section{APPLICABILITY OF ICHD-3 CRITERIA}

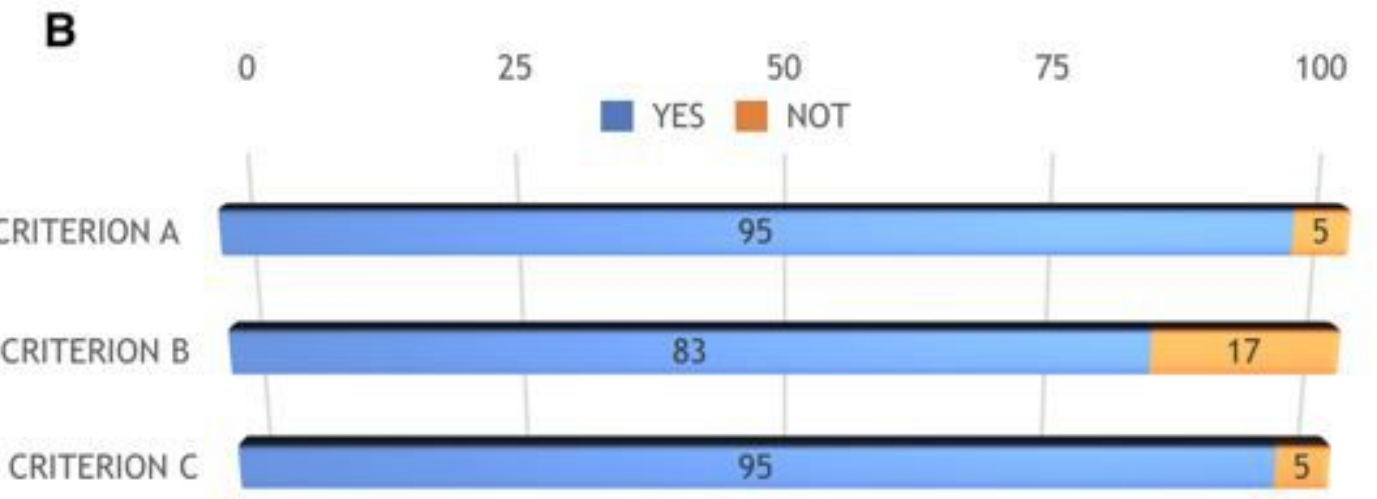

\section{Figure 2}

Applicability of ICHD-2 (2A) and ICHD-3 (2B) diagnostic criteria for $\mathrm{MOH}$. 